\title{
Finite-Element Methods for Singularly Perturbed Elliptic Boundary Value Problems and its Application to the Stationary Navier-Stokes Equations ${ }^{1}$ )
}

\author{
H. Goering and L. Tobiska
}

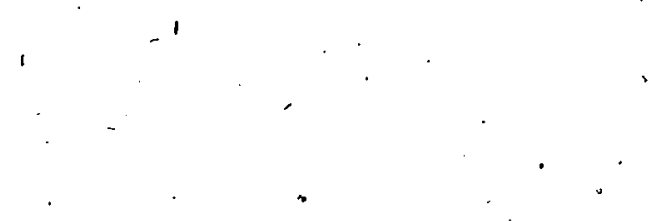

Wir betrachten einige Varianten der Methọde der finiten Elemente zur Lösung singulär gestörter elliptischer Randwertaufgaben 2. Ordnung, wobei das reduzierte Problem von erster Ordnung ist. Stabilitäts- und Konvergenzeigenschaften dieser Methoden und ihre Anwendung auf die stationären Navier-Stokes-Gleichungen für große Reynolds-Zahlen werden untersucht.

Мы рассматриваем некоторые варианты метода конечных элементов для решения сингулярно возмущённых зллиптических граничных задач второго порядка,' при которых рспуцированная задача - проблема первого порядка. Исследуются сходимость и устойчивость тӓких методов и их применение иа стационарное уравнение Навье-Стокса при больших чисел Рейнолдса.

We consider some modifications of finite-element methods for solving singularly perturbed elliptic beoundary value problems of second order where the reduced problem is of first order. Stability and convergence properties of such methods and the application to the stationary Navier-Stokes próblem for high Reynolds numbers are studied.

\section{Introduction}

For solving singularly perturbed elliptic boundary value problems, the common finite-element methods using, for instance, piecewise linear functions, are unstable and exhibit spurious oscillations unless the discretization parameter is not sufficiently small. Moreover, the occurrence of boundary layers influences the approximation properties in a negative sense - the usual error estimates become meaningless. For this reason, in the last years many modifications of the standard finiteelement method have been developed to overcome these difficulties, i.e. to guarantee stability and to obtain a good approximation of the exact solution. Such proposals are Petrov-Galerkin methods with piecewise polynomial bases [9], special integration rules for the convective terms [5], the utilization of special directional derivatives [3, 25], some mixed finite-element methods [13], the symmetrization of the bilinear form [2], the use of artificial diffusion [15, 16], asymptotically fitted methods $[8,22]$, hybrid upwind finite-element methods $[12,21,26]$ and the streamline diffusion method $[10,11,19]$.

Nowadays, from a mathematical point of view the last three methods are the best ones for solving singularly perturbed problems because of its mathematical foundations and its favourable properties. In the following we will give an overview on the main ideas and results of these three classes of methods in the case of the two-dimensional stationary convection-diffusion problem $\left(\Omega \subset \mathbf{R}^{2}, \Gamma=\partial \Omega\right)$

$$
-\varepsilon \triangle u+b(x) \nabla u+c(x) u=f(x) \text { in } \Omega, \quad u=0 \text { on } \Gamma,
$$

1) Vortrag auf der gemeinsamen Tagung vom 9:-13. 11. 1987 in Berga (DDR) der HFR Analysis und HFR Numerisehe Mathematik. 
and discuss'its application to the stationary Navier-Stokes equations

$$
-\varepsilon \triangle u+u \nabla \dot{u}+\nabla p=f \text { and } \operatorname{div} u=0 \text { in } \Omega, \quad u=0 \text { on } \Gamma .
$$

We will assume that $\Omega$ is a polygon, the functions $b, c$ and $f$ are sufficiently regular and $\varepsilon$ is a small positive parameter. Furthermore, we denote, by $|\cdot|_{k, p},\|\cdot\|_{k, p}$ the usual seminorm and norm in the Sobolev space $W^{k, p}(\Omega)$, by $(\cdot, \cdot)$ the scalar product in the space $L^{2}(\Omega)$ and by $C$ a generic constant independent of $\varepsilon$ and $h$.

\section{Asymptotically fitted finite-element methods}

The weak formulation of problem (1.1) reads as follows:

Find $u \in H_{0}{ }^{1}(\Omega)$ such that for all $v \in H_{0}{ }^{1}(\Omega)$

$$
B(u, v) \equiv \varepsilon(\nabla u, \nabla v)+(b \nabla u+c u, v)=(f ; v) .
$$

Under the assumption $\left(c-2^{-1} \operatorname{div} b\right)(x) \geqq \alpha>0$ for all' $x$ the bilinear form $B$ is $H_{0}{ }^{1}$-elliptic and by means of Lax-Milgram's theorem we obtain the existence of a unique solution $u$ of the problem (2.1). Choosing a conforming finite-element space $V_{h} \subset H_{0}{ }^{1}(\Omega)$ which we will specify later we get the following discrete problem:

Find $u_{h} \in V_{h}$, such that for all $v \in V_{h}$

$$
B\left(u_{h}, v\right)=(f, v) \text {. }
$$

Under the above assumption also problem (2.2) has a unique solution $u_{h}$. In order to obtain error estimates with $\varepsilon$-independent error constants, we will use the $\varepsilon$-weighted $H^{1}$-norm and the fitted norm defined by

$$
\|v\|_{c}=\left(\varepsilon\|v\|_{1,2}^{2}+.\|v\|_{0.2}^{2}\right)^{1 / 2} \quad \text { and } \cdot\|v\|=\|v\|_{\varepsilon}+\operatorname{sip}_{0 \neq v \in V} \frac{B(v, v)}{\|v\|_{\varepsilon}}
$$

respectively, which are equivalent to the $H^{1}$-nórm for fixed $\varepsilon>0$. Moreover, it holds $\|u\|_{0,2} \leqq\|u\|_{\varepsilon} \leqq\|u\|\|\leqq\| u \|_{1,2}$. From the $H_{0}{ }^{1}$-ellipticity of $B$ in the $\varepsilon$-norm with the constant $c_{1}=\min (1, \alpha)$ we have for each $w_{h} \in \dot{V}_{h}$

and conclude

$$
\left\|w_{h}-u_{h}\right\|_{\varepsilon} \leqq \frac{1}{c_{1}} \frac{B\left(w_{h}-u_{h}, w_{h}-u_{h}\right)}{\left\|w_{h}-u_{h}\right\|_{\varepsilon}} \doteq \frac{1}{c_{1}} \frac{B\left(w_{h}-u, w_{h}-u_{h}\right)}{\left\|w_{h}-u_{h}\right\|_{e}} .
$$

$$
\left\|u \doteq u_{h}\right\|_{e} \leqq\left\|u-w_{h}\right\|_{e}+\left\|\ddot{\|} w_{h}-u_{h}\right\|_{\varepsilon} \leqq\left\|u-w_{h}\right\|_{\varepsilon}+\frac{1}{c_{1}} \sup _{v \in V_{h}} \frac{B\left(w_{h}-u, \dot{v}\right)}{\|v\|_{e}} .
$$

Thus, the estimation of the error is reduced to the estimation of the approximation error in the fitted norm $\left\|u-u_{n}\right\|_{\varepsilon} \leqq C$ inf $\left\{\|\| u-u_{h} \| l: w_{h} \in V_{h}\right\}$. Usually, the approximation error is replaced by the interpolation error such that for instance for spaces of bilinear elements the estimate inf $\left\{\left|\left\|u-w_{h}\right\|\right|: w_{h} \in V_{h}\right\} \leqq C h|u|_{\mathbf{2}, 2}$ holds. Now, the occurence of boundary layers in the solution affects the boundedness of $|u|_{2,2}$ for $\varepsilon \rightarrow 0$, i.e. only $\varepsilon^{3 / 2}|u|_{2,2}$ is uniformly $\varepsilon$-bounded and the resulting estimate reads

$$
\left\|u-u_{h}\right\|_{\varepsilon} \leqq C h \varepsilon^{-3 / 2} \text {. }
$$

It is clear that this becomes meaningless provided that $\varepsilon \ll h$ : 
The principle of asymptotically fitted finite-element methods consists of splitting up the approximation error in two parts

$$
\inf _{w_{n} \in V_{h}}\|\| u-w_{B}\left\|\left|\leqq\|\| u-u_{a s}\left\|\mid+\inf _{w_{A} \in V_{n}}\right\| u_{a s}-w_{h}\|\|,\right.\right.
$$

namely in the asymptotic error $\mid\left\|u-u_{a}\right\| \|$ and the approximation error for the asymptotic solution $u_{a s}$. Thus, the investigation consists of two steps:

(i) Studying the structure of the asymptotic solution $u_{a s}$ and estimating the difference to the exact solution in the fitted norm.

(ii) Fitting the finite-element space $V_{h}$ according to the structure of $u_{a s}$ and estimating the approximation error.

A detailed treatment of the asymptotic behaviour and of the construction of asymptotic solutions $u_{a}$ in the maximum norm can be found in [7]. Let us consider the special case

$$
\Omega=(0,1) \times(0,1), b=\left(b_{1}, b_{2}\right) \text { with } b_{i}(x)>0, \therefore i=1,2
$$

in which the asymptotic approximation $u_{a s}$ consists of the solution $u_{0}$ of the reduced problem $\left(\Gamma_{-}=\{x \in \Gamma: b(x) \cdot n(x)<0, n\right.$ outer normal $)$

$$
b(x) \nabla u_{0}+c(x) u_{0}=f(x) \text { in } \Omega, u_{0}=0 \text { on } \Gamma_{-}^{-},
$$

two ordinary boundary layer terms

$$
\begin{gathered}
v_{1}(x)=-u_{0}\left(1, x_{2}\right)\left[\exp \left(-\frac{b_{1}\left(1, x_{2}\right)}{\varepsilon}\right)\left(1-x_{1}\right)-\left(1-x_{1}\right) \exp \left(-\frac{b_{1}\left(1, x_{2}\right)}{\varepsilon}\right)\right], \\
\cdots \\
v_{2}(x)=-u_{0}\left(x_{1} ; 1\right)\left[\exp \left(-\frac{b_{2}\left(x_{1}, 1\right)}{\varepsilon}\right)\left(1-x_{2}\right)-\left(1-x_{2}\right) \exp \left(-\frac{b_{2}\left(x_{1}, 1\right)}{\varepsilon}\right)\right]
\end{gathered}
$$

and a corner layer term

$$
v_{3}(x)=u_{0}(1,1) \prod_{i=1}^{2}\left[\exp \left(-\frac{b_{i}(1,1)}{\varepsilon}\right)\left(1-x_{i}\right)-\left(1-x_{i}\right) \exp \left(-\frac{b_{i}(1,1)}{\varepsilon}\right)\right]
$$

In [22] the estimate

$$
\left\|u-u_{a s}\right\| \| \leqq C \varepsilon^{1 / 2}
$$

was proven where $u_{a s}=u_{0}+v_{1}+v_{2}+v_{3}$. It should be mentioned that the proof of (2.7) is nonstandard because the solution $u_{0}$ of the reduced problem ,belongs only to the space $C(\bar{\Omega}) \cap W^{1, \infty}(\Omega)$.

Now we have to choose the finite-element space $V_{h}$ in such a way that the interpolation error becomes small. On a square mesh of fineness $h=1 / N$ we add the conumon bilinear functions defined by

$$
\varphi_{i j}(x)=\varphi_{i}\left(x_{1}\right) \varphi_{j}\left(x_{2}\right), \quad \varphi_{i}(t)=\left\{\begin{array}{l}
\frac{t}{h}-i+1, t \in[(i-1) h, i h], \\
i+1-\frac{t}{h}, t \in[i h,(i+1) h], \\
0, \text { otherwise. }
\end{array}\right.
$$


The following functions fitting the boundary layer terms (2.4)-(2.6):

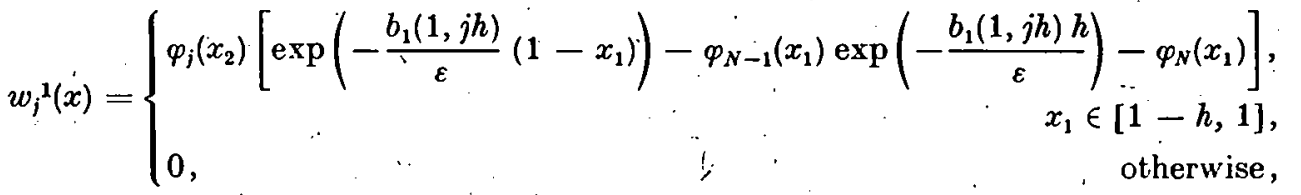

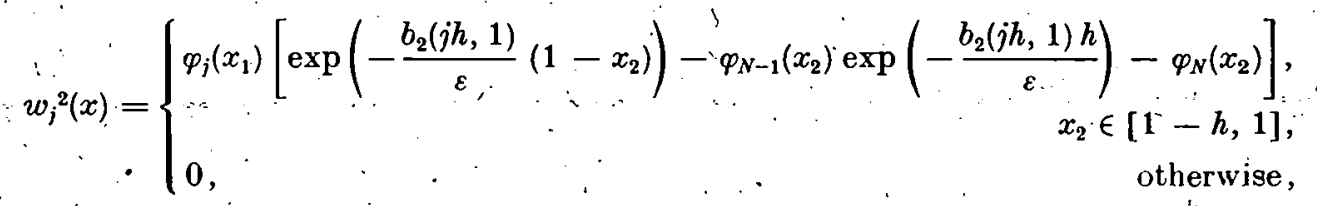

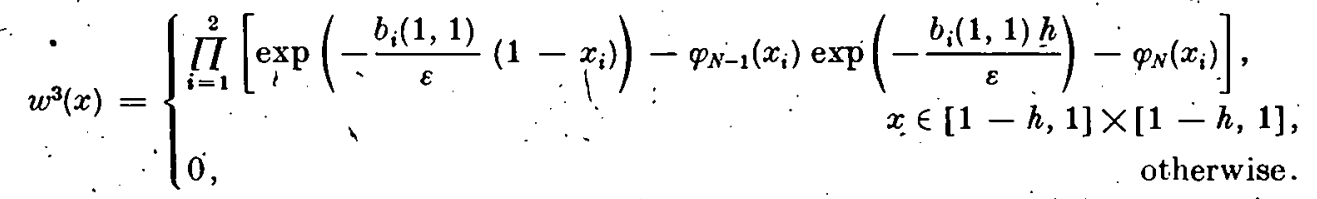

Now, the asymptotically fitted finite-element method is characterized by (2.2) with $V_{h}=\operatorname{span}\left(\varphi_{i j}, w_{j}{ }^{1}, w_{j}{ }^{2}, w^{3}: i, j=1, \ldots, N-1\right)$. In [22] the interpolation error was estimated by

$$
\inf _{w_{n} \in V_{n}}\left\|u_{a s}-w_{h}\right\| \leqq C\left(\varepsilon+h^{1 / 2}\right)+C(m)(\varepsilon / h)^{m}
$$

with $m>0$, arbitrary. The valve $1 / 2$ of the $h$-exponent is due to the fact that $u_{0}$ is not sufficiently regular.

Theorem.1: Let $\bar{u}_{h}$ be the solution of the discrete problen (2.2) without fitting. if $\varepsilon \geqq c h^{1 / 2}$ and with the above mentioned fitting if $\varepsilon \leqq c h^{1 / 2}$. Then, inder the assumption (A1) we have the error estimate

$$
\left\|u-\tilde{u}_{h}\right\|=C h^{1 / 4}
$$

uniformly with respect to $\varepsilon$.

Proo : Combining the estimates (2.3), (2.7), and (2.8) we obtain (2.9)

In [22] asymptotically fitted finite-element methods for some other cases have been derived and error estimates have been given. The main advantage of asy mptotically fitted finite-element methods consists of the favourable approximation-properties within the layers which is based on the relatively large amount of analytical a-priori knowledge. 'Thus, this method can' be used if some information on the position and the structure of boundary layers are known. In numerical experiments it was demonstrated that the method is stable and does not produce any oscillations in the solution.

\section{Hybrid upwind finite-element methods}

The main objective of hybrid upwind finite-element methods is to conserve the inverse monotonicity of the continuous problem which can be established if

$$
c(x) \geqq c_{0} \geqq 0 \text { in } \Omega
$$

is fulfilled. As a consequence stability of the discrete problem in $L^{\infty}$-norms can be shown. 
Let $\mathfrak{T}_{b}$ be an admissible, regular triangulation of weakly acute type, i.e. all interior angles are smaller or equal to $\pi / 2$, and. let $\left\{B_{i}\right\}$ be the set of nodal points. On each triangle $K$ we use linear functions, i.e. we set $V_{h}=\left\{v_{h} \in C(\bar{\Omega}):\left.v_{h}\right|_{K} \in P_{1}(K), v_{k} \mid r\right.$ $=0\}$. Let $\left\{\Phi_{i}\right\}$ be a basis of $V_{. h}$ with $\Phi_{i}\left(B_{j}\right)=\delta_{i j}$. Then, the discretization of $\varepsilon(\nabla u, \nabla v)$ corresponds to an $M$-matrix but in general we have positive outer diagonal elements from $(b \nabla u+c u, v)$. In order to modify the standard finite-element method we consider a secondary decomposition of $\bar{\Omega}$ into dual domains $\left\{D_{i}\right\}$ where $D_{i}$ denotes that polygon whose vertices are circumcentres of triangles surrounding $B_{i}$. Furthe! more, let $A_{i}$ be the set of indices $j$ such that $B_{i}$ and $B_{i}$ are neighbour nodes, $B_{i j}$ the midpoint of the side $B_{i} B_{j}$ and $\Gamma_{i j}$ the side of $\partial D_{i}$ passing $B_{i j}$. We also need the characteristic function $\Phi_{i}$ of the domain $D_{i}$ and introduce the lumping operator from $\dot{C}(\bar{\Omega})$ into $L^{2}(\Omega)$ defined by $v \rightarrow \bar{v}_{h}=\sum_{i} v\left(B_{i}\right) \bar{\Phi}_{i}$. We denote by $n_{i j}$. the unit outer normal vector on the part $\Gamma_{i j}$ of the boundary $\partial D_{i}$ and by $\beta_{i j}$ an approximation of $\int_{\Gamma_{s}} b \cdot n_{i j} d s$. Now, the hybrid"upwind finite-element method is given as follows:

Find $u_{h} \in V_{h}$ such that' for all $v_{h} \in V_{h}$

where

$$
B_{h}\left(u_{h}, v_{h}\right) \equiv \varepsilon\left(\nabla u_{h}, \nabla v_{h}\right)+b_{h}\left(u_{h}, v_{h}\right)+\left(\overline{c u}_{h}, \bar{v}_{h}\right)=\left(f, \bar{v}_{h}\right),
$$

$b_{h}\left(u_{h}, v_{h}\right)=\sum_{i} \dot{v}\left(P_{i}\right) \sum_{j \in A_{i}} \beta_{i j}\left(\lambda_{i j}-1\right)\left[u_{h}\left(B_{i}\right)-u_{h}\left(B_{j}\right)\right], \quad \lambda_{i j}= \begin{cases}1, & \beta_{i j} \geqq 0, \\ 0, & \beta_{i j}<0 .\end{cases}$

Using the above mentioned basis $\left\{\Phi_{i}\right\}$ it is easy to see that the matrices corresponding to $b_{h}\left(u_{h}, v_{h}\right)$ and $\left(\bar{c} \bar{u}_{h}, \bar{v}_{h}\right)$ have nonpositive outer diagonal elements and, by means of a chạin property, we can establish that the system matrix of (3.1) is an $M$-matrix. Consequently, the discrete problem is inverse monotone.

In order to formulate the convergence properties of the method we use the same norms as in Section 2 . Let $\beta_{i j}$ be calculated by the midpoint rule applied to $\int_{\Gamma_{i j}} b \cdot \dot{n_{i j}} d s$.

Then we obtain the $V_{h}$-ellipticity of $B_{h}$ and, by a modification of the first lemma of Strang, the estimate

$\left\|u-u_{h}\right\|_{i} \leqq C \inf _{v_{A} \in V_{A}}\left(\left\|u-\dot{v}_{h}\right\|_{i}+\sup _{w_{A} \in V_{A}} \frac{B\left(v_{h}, w_{h}\right)-B_{h}\left(\dot{v}_{h}, w_{h}\right)}{\left\|w_{h}\right\|_{c}}+\sup _{w_{h} \in V_{h}} \frac{\left(f, w_{h}-\bar{w}_{h}\right)}{\left\|w_{h}\right\|_{\varepsilon}}\right)$

On the basis of this the estimates

$$
\left\|u \div u_{h}\right\|_{\varepsilon} \leqq \begin{cases}\dot{C} h \varepsilon^{-1 / 2}|u|_{2,2} & \text { for an arbitrary mesh, } \\ C h|u|_{2,2} & \text { for a regular mesh }\end{cases}
$$

, were proven in [21]: Here a mesh is called regular if it is built from three families of parallels. Using a lemma from Stampaccia, it is also possible to obtain the $L^{\infty}$-estimates

$$
\left\|u-u_{h}\right\|_{0, \infty} \leqq \begin{cases}C(\sigma) h^{\sigma} \varepsilon^{-1 / 2}|u|_{2, \infty} & \text { fór an àrbitrary mesh, } \\ C(\sigma) h^{\sigma}|u|_{2, \infty} & \text { for a regular mesh }\end{cases}
$$

with $\sigma \in(0,1)$, arbitrary. Because the seminorms $|u|_{2,2}$ and $|u|_{2, \infty}$ are not uniformly bounded with respect to $\dot{\varepsilon}$, the estimates (3.3) and (3.4) become meaningless if $\varepsilon$ tends to zero. However, Risch [21] was sucessful, in proving local estimates in subdomains where no boundary layers occur (called global domain in the notation of [7]). For the special case

$$
\Omega=(0,1) \times(0,1), b=\left(b_{1}, 0\right) \text { with } b_{1}>0, \mathfrak{T}_{n} \text { a regular mesh, }
$$


we have boundary layers of ordinary and parabolic type near $\dot{x_{1}}=1, x_{2}=0, x_{2}=1$ and corner layers of different type in the vertexes of $\Omega$.

The,orem 2: Let the assumptions (A 2), (A 3) be fulfilled and the distants $d, d_{1}$ and $d_{2}$ be defined: by $d=\max (\dot{\varepsilon}, h), d_{1}=O\left(d^{1 / 2}|\ln d|\right), d_{2}=O(d|\ln d|)$. Then, in the global domain $\Omega^{*}=\left[0,1-d_{2}\right] \times\left[d_{1} ; 1-d_{1}\right]$ we have the local estimates

$$
\left\|u-u_{h}\right\|_{e, \Omega *} \leqq C h, \quad \cdot\left\|u-u_{h}\right\|_{0, \infty, \Omega^{*}} \leqq C(\sigma) h^{\sigma}, \quad \sigma \in(0,1) \text { arbitrary. }
$$

For the proof see the more complex cases considered in [21]

The main advantages of the hybrid upwind finite-element methods consist in the good stability properties (inverse monotonicity of the discrete problem) combined with localization properties which do not require any a-priori knowledge on the position of boundary layers. We mention that in [21] also the case of a.system of : equations of the form (1.1) was studied.

\section{Streàmline diffusion method}

The mathematical foundation of the streamline diffusion method was given by NĀVERT [19]: The method combines high order of convergence with good stability properties. In order to sketch the procedure we start with the variational form of (1.1):

Find $u \in H_{0}{ }^{1}(\Omega)$ such $^{2}$ that for. all $v \in H_{0}{ }^{1}(\Omega)$

$$
B(u, v) \equiv \varepsilon(\nabla u, \nabla v)+(b \nabla u+c u, v)=(f, v) .
$$

Provided that the exact solution belongs to $H^{2}(\Omega)$, for all $v \in H_{0}{ }^{1}(\Omega)$ the relation $(-\varepsilon \triangle u+b \nabla u+c u, b \nabla v)=(f, b \nabla v)$ is satisfied such that each solution $u \in H_{0}{ }^{1}(\Omega)$ $\cap H^{2}(\Omega)$ of (2.1) fulfils

$$
B_{\delta}(u, v) \equiv(-\varepsilon \delta \triangle u, b \cdot \nabla v)+\varepsilon(\nabla u, \nabla v)+(b \nabla u+c u, v+\delta b \nabla v)=(f, v+\delta b \nabla v)
$$

for all $v \in H_{0}^{1}(\Omega)$ : We use piecewise polynomials of degree $k$, that means we set $V_{h}=\left\{v_{h} \in C(\bar{\Omega}):\left.v_{h}\right|_{K} \in P_{k}(K),\left.v_{h}\right|_{\Gamma}=0\right\}$. Then, the following discrete problem is derived from (4.1):

Find $u_{h} \in V_{h}$ such that for all $v_{h} \in V_{h}$,

$$
B_{h}\left(u_{h}, v_{h}\right)=\left(f, v_{h}+\delta b: \nabla v_{h}\right)
$$

where the bilinear form $B_{h}$ is defined by

$$
\begin{aligned}
B_{h}\left(u_{h}, v_{h}\right) \equiv & -\varepsilon \delta \sum_{K}\left(\triangle u_{h}, b \cdot \nabla v_{h}\right)_{K}+\varepsilon\left(\nabla u_{h}, \nabla v_{h}\right) \\
& +\left(b \nabla u_{k}+c u_{h}, v_{h}+\delta b \cdot \nabla v_{h}\right) .
\end{aligned}
$$

We remark that for $\delta=0(4.2)$ is equal to the standard Galerkin finite-element method.

We introduce a fitted norm in $H_{0}^{1}(\Omega)$ defined by $\|u\|=\left(\varepsilon|u|_{1,2}^{2}+\delta\|b \cdot \nabla u\|_{0,2}^{2}\right.$ $\left.+\alpha\|u\|_{0,2}^{2}\right)^{1 / 2}$ where $\alpha$ fulfils $\left(c-2^{-1} \operatorname{div} b\right)(x) \geqq \alpha>0$ for all $x \in \Omega$. By means of inverse inequalities for estimating of $\sum_{K}\left(\triangle u_{h}, b \cdot \nabla u_{h}\right)_{K}$, we can show the $H_{0}{ }^{1}$-ellipticity of $B_{h}$ in the fitted norm provided $\delta$ is sufficiently small. To be more specific, we 
have for $0 \leqq \delta \leqq \min \left(\delta_{0}, c_{1} \cdot h^{2} \varepsilon^{-1}\right)$ the estimate .

$$
B_{h}\left(u_{h}, u_{h}\right) \geqq C_{2} \mid\left\|u_{h}\right\|^{2} \quad \text { for all } u_{h} \in H_{0}^{1}(\Omega), C_{2}>0 \text {. }
$$

Taking into consideration that

?. $\quad\left|\left(f, v_{h}+\delta b \cdot \nabla v_{h}\right)\right| \leqq\|j\|_{0.2}\left(\left\|v_{h}\right\|_{0.2}{ }^{-}+\delta\left\|b \cdot \nabla v_{h}\right\|_{0.2}\right) \leqq C_{3}\|f\|_{0,2} \cdot\left\|v_{h}\right\| \|$

we obtain.from (4.3) a stability result in the form $\left.\mid\left\|u_{h}\right\|\right\rfloor \leqq C\|f\|_{0,2}$.

The good stability results observed in numerical test problems are due to the term $\delta\|b \cdot \nabla u\|_{0.2}^{2}$ contained in the fitted norm defined above. As for as the inverse monotonicity of the discrete problem is concerned it is easy to see that the system matrix corresponding to (4.2) in general is not an $M$-matrix. For example, in the particular case that $b=$ const, $c=0, \Omega=(0,1) \times(0,1)$, piecewise linear elements are used and the triangulation is of Friedrichs-Keller type, the nonnegativity of the outer diagonal elements can not.be fulfilled for sufficiently small $\varepsilon$ compared with $h$. Moreover, numerical test examples permit the conclusion that the discrete problem is in fact not inverse monoton for $\varepsilon$ tending to zero.

By carefully handling in majorizing the bilinear form $B_{h}$ Nävert was able to prove error estimates.

Theorem 3: Let $\varepsilon<h$ and $\delta=c_{1} h$. Then we have the error estimate

$$
\left|\left\|u-u_{h}|\||=C h^{k+1 / 2}|u|_{k+1,2}\right.\right.
$$

for piecewise polynomials of degree $k$.

However, the seminorm $|u|_{k+1.2}$ on the right-hand side of (4.4) is not uniformly bounded with respect to $\varepsilon$. Therefore, local estimates in domains without boundary layers are more important.

For the case (A3) already studied in Theorem 2 in frame of hybrid upwind finiteelement methods, the results of [19] yield the estimate \|\|$u-u_{h} \|_{\Omega^{*}} \leqq C h^{k+1 / 2}$, $\varepsilon<h$.

Comparing the streamline diffusion method with the asymptotically fitted method and with the hybrid upwind method we observe that it represents an intermediate position in some sense. The asymptotically fitted methods guarantee $\varepsilon$-uniform convergence but stability is only obtained in a rather weak sense. The streamline diffusion method on the one hand yields better stability properties but on the other hand it does not guarantee $\varepsilon$-uniform convergence. However, the local estimates of high order of convergence show the capability of the method. Finally, the hybrid upwind finite-element method gives stability in $L^{\infty}$-norms and preserves the inverse monotonicity of the problem to be solved. But, in contrary to the streamline diffusion. method, it only works for linear elements.

\section{Application to the stationary Navier-Stokes equations}

The stationary Navier-Stokes problem consists in determining the velocity $u$ and the .pressure $p$ of a fluid which are solutions of the system of equations $\left(\Omega \subset \mathbf{K}^{2}, \Gamma=\partial \Omega\right)$

$$
-{ }^{\prime} \triangle \Delta u+(u \nabla) u+\nabla p=f \text { and } \operatorname{div} \cdot u=0 \text { in } \Omega, u=0 \text { on } \Gamma \text {. }
$$

Multiplying these equations by functions belonging to $V=H_{0}{ }^{1}(\Omega)^{2}$ and $Q=L_{0}{ }^{2}(\Omega)$ $=\left\{q \in L^{2}(\Omega): \int_{\Omega} q d x=0\right\}$, respectively, and integrating by parts we obtain the variational formulation in the primitive variables: 
Find $(u, p) \in V \times Q$ such that

$$
\begin{array}{ll}
\varepsilon a(u, v)+b(u, u, v)-(p, \operatorname{div} v)=(f, v) & \forall v \in V, \\
(q, \operatorname{div} u)=0 & \forall q \in Q,
\end{array}
$$

where we used the notations

$$
a(u, v)=\int_{\Omega} \nabla u \nabla v \dot{d} x, \quad b(u, v, w)=\frac{1}{2} \int_{\Omega}(u \nabla v w-u \nabla w v) d x
$$

It is well known that the problen (5.1) admits at least one solution and that this solution is unique provided that $\varepsilon \cdot>\varepsilon_{0}\left(\|f\|_{0.2}, \Omega\right)$ [6]. It shiould be mentioned that stability problems which are similar to the case of convection-diffusion equations arise, for small $\varepsilon$ (large Reynold numbers) already before the bound $\varepsilon_{0}$ is attained.

Because of the lack of sufficient a-priori knowledge concerning the asymptotic behaviour of the solutions of (5.1) for sniall values of $\varepsilon$, we shall not discuss asymptotically fitted methods in this section.

At first we consider a finite-element method of hybrid upwind type. To this end we start with a pair of finite-element spaces $V_{h}, Q_{h}$ satisfying the discrete version of the Ladyzhenskaya-Babuska-Brezzi condition

$$
\sup _{v_{h} \in V_{h}}\left(q_{h}, \operatorname{div} v_{h}\right) /\left|v_{h}\right|_{1,2} \geqq \beta\left\|q_{h}\right\|_{0,2} \quad \text { for all } q_{h} \in Q, \beta>0
$$

which is very important for deriving convergence results. Let $\Omega$ be a polygon divided into triangles $K$. We denote by $B_{\text {j }}$ the midpoints of edges and define the finiterelement spaces by

$$
\begin{aligned}
& V_{h}=\left\{v_{h}:\left.v_{h}\right|_{K} \in P_{1}(K)^{2}, v_{h} \text { cont. in } B_{j}, v_{h}\left(B_{j}\right)=0 \text { if } B_{j} \in \Gamma\right\}, \\
& Q_{h}=\left\{q_{h} \in Q:\left.q_{h}\right|_{K} \in P_{0}(K)\right\} .
\end{aligned}
$$

Because the discrete velocity space $V_{h}$ consists of piecewise linear functions which on the edges are continuous only in the midpoints, $V_{b}$ is not contained in $K$ (nonconforming finite-element methods) and we have to:extend the bi- and trilinear form in (5.1). 'This can be done in a natural way by an elementwise calculation of the corresponding integrals. Iet us introduce the meshdependent norm

$$
\|u\|_{h}=\left(\sum_{K} \int_{K}(\nabla u)^{2} d x\right)^{1 / 2} \quad \text { for } u \in V+\dot{V}_{h} .
$$

The standard finite-element method studied in $[4,27]$ reads as follows:

$$
\begin{aligned}
& \text { Find }\left(u_{h}, p_{h}\right) \in V_{h} \times Q_{h} \text { such that } \\
& \varepsilon a\left(u_{h}, v_{h}\right)+b\left(u_{h}, u_{h}, v_{h}\right)-\left(p_{h}, \operatorname{div} v_{h}\right)=\left(f, v_{h}\right) \quad \forall v_{h} \in V_{h}, \\
& \left(q_{h}, \operatorname{div} u_{h}\right)=0 \quad \forall q_{h} \in Q_{h}:
\end{aligned}
$$

-It converges of order one: Applying a hybrid upwind method to (5.1), we have only' to change the discretization of the convective term with the aim of a better reflection. of the dominate influence of the convective term for small values of $\varepsilon$. For this, following the idea of $\mathrm{OH}_{\mathrm{O}} \mathrm{R}$ and UsHIJIMA [20] we define a secondary decomposition of $\Omega$ into domains $D_{i}$. Each inner node $B_{i}, i \doteq 1, \ldots, N$, corresponds to a dual domain $D_{i}$ which is defined by the barycenters $S_{1}, S_{2}$ of the neighbouring triangles $K_{1}, K_{2}$ of $B_{i}$. Furthermore, let $A_{i}$ be the set of indices $j$ such that $B_{i}$ and $B_{j}$ are neighbour nodes and $n_{i j}$ be the unit outer normal vector with respect to $D_{i}$ along the part $\Gamma_{i j}$ 
of $\partial D_{i}$ between $B_{i}$ and $B_{j}$. Now, we can derive the following upwind discretization $b_{h}$ of the trilinear form $b$ :

$$
b_{h}(u, v, w)=\sum_{i=1}^{N} \sum_{j \in \Lambda_{i}} \int_{\Gamma_{i j}} u n_{i j} d s^{\prime}\left(1-\lambda_{i j}(u)\right)\left[v\left(B_{j}\right)-v\left(B_{i}\right)\right] w^{\prime}\left(B_{i}\right),
$$

where $\lambda_{i j}(u)$ clepends on the flux through $\Gamma_{i j}$ according to

$$
\lambda_{i j}(u)= \begin{cases}1 & \text { if } \int_{\Gamma_{i j}} u n_{i j} d s \geqq 0 \\ 0 & \text { otherwise. }\end{cases}
$$

Our hybrid upiwind finite-element method for solving the stationary Navier-Stokes problem (5.1) is characterized as follows:

Find $\left(u_{h}, p_{h}\right) \in V_{h} \times Q_{h}$ such that

$$
\begin{aligned}
& \varepsilon a\left(u_{h}, v_{h}\right)+b_{h}\left(u_{h}, u_{h}, v_{h}\right)-\left(p_{h}, \operatorname{div} v_{h}\right)=\left(f, v_{h}\right), \quad \forall v_{h} \in, V_{h}, \\
& \left(q_{h}, \operatorname{div} u_{h}\right)=0
\end{aligned}
$$

Theorem 4: The discrete problem (5.6) admits at least one solution $\left(u_{h}, p_{h}\right) \in V_{h} \times Q_{h}$ .which is unique, provided $\varepsilon>\varepsilon_{0}(h)$ where $\varepsilon_{0}(h) \rightarrow \varepsilon_{0}$ as $h \rightarrow 0$. Moreover, for $\varepsilon>\dot{\varepsilon}_{0}(h)$ the error estimate

$$
\begin{aligned}
& \left\|u-u_{h}\right\|_{h}+\left\|p-p_{h}\right\|_{0,2} \leqq C(\sigma) h^{1-\sigma}, \quad \sigma>0 \text { arbitrary, } \\
& \text { is satisfied if }(\dot{u}, p) \in H^{2}(\Omega)^{2} \times H^{1}(\Omega) .
\end{aligned}
$$

The crucial point in proving Theorem 4 is that the function $u$ in (5.5) contrary to the function $b$ in (1.1) is not sufficiently regular since it only belongs to the space $V_{h}$. For details we refer to [23], numerical test examples can be found in [24]. A further advantage of the proposed methods consists in a favourable property of the linear systems of equations if they are generated by a fixed point procedure. Namély, let $\left\{\left(\Phi_{i}, 0\right)\right\},\left\{\left(0, \Phi_{i}\right)\right\}$ be a basis of $V_{h}$ defined by $\Phi_{i}^{\prime}\left(B_{j}\right)=-\delta_{i j}$. Then, for fixed $z \in V_{h}$ the matrix corresponding to $\varepsilon a\left(u_{h}, v_{h}\right)+b_{h}\left(z, u_{h}, v_{h}\right)$ is an $M$-matrix, provided the mesh is of weakly acute type [23].

Finally, we discuss a nonconforming streamline diffusion method for the stationary Navier-Stokes problem. We will use the same finite-element spaces $V_{h}, Q_{h}$ defined by (5.2), (5.3) and start, with the standard finite-element method (5.4). Provided that the exact solution $(u, p)$ belongs to the space $H^{2}(\Omega)^{2} \times H^{1}(\Omega)$ we obtain by testing the relation $-\varepsilon \triangle u+(u \nabla) u+\nabla p=f$ on each element with $\delta u_{h} \nabla v$.

$-\varepsilon \delta \sum_{K}\left(\triangle u, u_{h} \nabla v\right)_{K}+\delta \sum_{K}\left(u \nabla u, u_{h} \nabla v\right)_{K}+\delta \sum_{K}\left(\nabla p, u_{h} \nabla v\right)_{K}=\delta \sum_{K}\left(f, u_{h} \nabla v\right)_{K}$

Since wé have piecewise linear elements for the velocity and piecewise constant elements for the pressiire, the discrete problem reduces to the following form:

Find $\left(u_{h}, p_{h}\right) \in V_{h} \times Q_{h}$ such that

$$
\begin{aligned}
& \varepsilon a\left(u_{h}, \dot{v}_{h}\right)+b\left(u_{h}, u_{h}, v_{h}\right)+\delta \sum_{K}\left(u_{h} \nabla u_{h}, u_{h} \nabla v_{h}\right)_{h}-\left(p_{h}, \operatorname{div} v_{h}\right) \\
& =\sum_{K}\left(f, v_{h}+\delta u_{h} \nabla v_{h}\right)_{K} \quad \text { for all } v_{h} \in v_{h}, \\
& \left(\dot{q}_{h}, \operatorname{div} u_{h}\right)=0 \quad \text { for all } q_{h} \in Q_{h} .
\end{aligned}
$$

The, additional term $\delta \sum\left(u_{h} \nabla u_{h}, u_{h} \nabla v_{h}\right)_{K}$ on the left-hand side of (5:7) has a stabilizing effect on the discrete problem for small $\varepsilon$. 
Theorem 5: Let $\delta$ satisfy $0 \leqq \delta \leqq C_{1} h^{1+\sigma}, \sigma>0$ arbitrary, and let the exact solution $(u, p)$ belong to $\left(W^{1, \infty}(\Omega) \times H^{2}(\Omega)\right)^{2} \times H^{1}(\Omega)$. Then there are constants $\varepsilon_{0}$ and $h_{0}$ such that for $\varepsilon>\varepsilon_{0}$ and $h \leqq h_{0}$ the problems (5.1) and (5.7) have unique solutions which satisfy the error estimate

$$
\varepsilon\left\|u-u_{h}\right\|_{h}^{2}+\sum_{K}\left\|u_{h} \cdot \nabla\left(u-u_{h}\right)\right\|_{0,2 . K}^{2} \leqq C h^{2}, \quad . \quad\left\|p-p_{h}\right\|_{0,2} \leqq C h .
$$

For the proof and further results concerning the case $\varepsilon>\varepsilon_{0}$ we refer to [18]

Comparing the streamline diffusion method with the hybrid upwind finite-element method we observe that, contrary to the linear case, the strong smoothness assump'tion $u \in W^{1, \infty}(\Omega)^{2}$ becomes necessary. First order of convergence can be established whereas the hybrid upwind method converges almost of first order. As far as the stability is concerned both methods achieve a stabilizing effect by the additional terms $b_{h}\left(u_{h}, u_{h}, u_{h}\right)$ and $\delta \sum_{\vec{K}}\left\|u_{h} \nabla u_{h}\right\|_{0.2, K}^{2}$ which are not identically zero on $V_{h}$ as in the standard finite-element method.

\section{REFERENCES}

[1] Barrett, K. E., and G. Demonski: .Finite element solutions of convective diffusion problems. Int. J. Num. Neth. Eng. 14 (1979), $1511-1524$.

[2] Barretr, J. W., and K. W. Morron: Optimal Petrov-Galerkin methods through approximate symmetrization. IMA J. Numer. Anal. 1 (1981), 439-468.

[3] Bristeau, M. O., et al: A finite element approximation of Navier-Stokes equations for incompressible viscous fluids. Iterative methods of solution. Lect. Notes Math. 771 (1980), 78-121.

[4] Crouzeix, M., and P.-A. Raviart: Conforming and nonconforming finite element methods for solving the stationary Stokes equations. Rev. Franc. Aut. Inf. Rech. Oper. Ser. Rouge 7 (1973) no. R-3, 33-75.

[5] Dervieux, A., and F. Thomasset: A finite element method for the simulation of a Rayleigh-Taylor instability. Lect. Notes Math. 771 (1980), 145-159.

[6] Grrault, V., and P.-A.'Raviakt: Finite Element Methods for Navier-Stokes Equations: Theory and Algorithms. Berlin-Heidelberg-New York-Tokyo: Springer-Verlag 1986.

[7] Goerriso, H., et al: Singular perturbed differential equations. Berlin: Akademie-Verlạg 1983.

[8] Groen, P. P. N.; and P. W. Hemker:-Error bounds for exponentially fitted Galerkin methods applied to stiff two-point boundary value problems; In: Numerical analysis of singular perturbation problems (eds: P. W. Hemker and J.J.H. Miller). London: Academic Press 1979.

[9] Hernrich, J. C., and O. C. Zienckiwicz: Quadratic finite element scheme for two-dimensional convective transport problems. Int. J. Num. Meth. Eng. 11 (1977), $1831-1844$.

[10] Hugnes; T.'J. R., and A. Brooks: A Theoretical Framework for Petrov-Galerkin Methods with Discontinuous Weighting Functions: Application to the Streamline Upwind Procedure. In: Finite Elements in Fiuids, Vol. 4 (Ed.: R. H. Gallagher). Baffin Lane-Chichester-Sussex : J. Wiley \& Sons Lim. 1982.

[11] Hughes, T. J. R., and M. MALLET: A new finite element formulation for computational fluid dynamics III. The generalised streamline operator for multidimensional advectivediffusive systems. Comput. Meth. Appl. Mech. Eng. 58 (1986), $305-328$.

[12] IKEDA, T.: Maximum principle in finite element models for convection-diffusion phenomena. Amsterdam - New York-Oxford: North-Holland Publ. Comp. 1983.

[13] Jafrre, J.: Approximation par une méthode d'élements finis mixtes d'une équation du type diffusion convection stationaire. Rapp: Rech. (LABORIA-INRIA) 367 (1979).

[14] Johnson, C., and J. Saranen:' Streamline diffusion methods for the incompressible Euler and Navier-Stokes equations. Math. Comp. 47 (1986) 175, 1-18. 


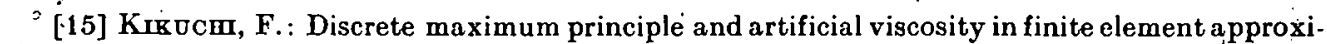
mations to convective diffusion. equations. Inst. Space Aeronaut. Sci. Rep. 550.(1977), $153-166$.

[16] Kik UCH, F., and T. UsmJima: On finite element methods for convection dominated phenomena. Math. Meth. Appl. Sci. 4 (1982), 98-122.

[17] Lesaint, P., and P.-A.RavLART: On a finite element method for solving the neútron transport equation, In: Mathematical Aspects of Finite Element Methods in Partial Differential Equations (Ed.: C. de Boor). New York: Academic Press 1974.

[18] LUBE, G., and L. Tobiska: A nonconforming finite element method of streamline diffusion type for the incompressible Navier-Stokes equations. Preprint Math. 4/87. Magdeburg (DDR): Techn. Univ. 1987.

[19] NävERT, U.: A finite element methöd for convection-diffusion problems. Dissertation. Göteborg: Chalmers Univ. Techn. 1982.

[20] Onmori, K., and T. Uswrma: A technique of upstream type applied to a linear nonconforming finite element approximation of convective diffusion equations. RAIRO, Anal. Numer. 18 (1984), 309-332.

[21] RIScr, U.: Ein hybrides upwind-FEM-Verfahren und dessen Anwendung auf schwach gekoppelte elliptische Differentialgleichungssysteme mit dominanter Konvektion. Dissertation. Magdeburg (DDR): Techn. Univ. 1986.

[22] ScruwEck, F.: Eine asymptotisch angepaßte Finite-Element-Methode für singulär gestörte elliptische Randwertaufgaben. Dissertation. Magdeburg (DDR): Techn. Univ. 1986.

[23] Schneweck, F., and L. Toвiska: A nonconforming finite clement method of upstream type applied to the stationary Navier-Stokes equations. Preprint Math. 2/87. Magdeburg (DDR): Techn. Univ. 1987.

[24] Schreweck, F., and L. TobISka: Eine upwind FEM zur Lösung des statiónären NavierStokes Problems Wiss. Z. Techn. Univ. Magdeburg (DDR) 31 (1987) 5, 73-76.

[25] TaвaTa, M.: Uniform convergence of the upwind finite element approximation for semilinear parabolic problems. J. Math. Kyoto Univ. 18 (1978), 327-351.

[26] TaBATA, M.: Conservative upwind finite element approximation and its applications. In: Analytical and Numerical Approaches to Asymptotic Problems in Analysis (Eds: S. Axelsson et al.). Amsterdam-New York-Oxford: North-Holland Publ. Comp. 1981.

[27] Temam, R.: Navier-Stokes Equations: Theory and Numerical Analysis. AmsterdamNew York-Oxford: North-Holland Publ. Co. 1979.

Manuskripteingang: 14.01 .1988

VERFASSER:

Prof. Dr. Herbert Goering und Doz. Dr. Lutz Tobiska

Sektion Mathematik

der Technischen Universität „Otto von Guericke“

PSF 124

DDR - 3010 Magdeburg 